\title{
ATLAS Superconducting Solenoid On-Surface Test
}

\author{
R. J. M. Y. Ruber, Y. Makida, L. Deront, Y. Doi, T. Haruyama, F. Haug, M. Kawai, T. Kondo, Y. Kondo, S. Mizumaki, \\ G. Olesen, O. V. Pavlov, M. Pezzetti, O. Pirotte, E. Sbrissa, H. H. J. ten Kate, and A. Yamamoto
}

\begin{abstract}
The ATLAS detector is presently under construction as one of the five LHC experiment set-ups. It relies on a sophisticated magnet system for the momentum measurement of charged particle tracks. The superconducting solenoid is at the center of the detector, the magnet system part nearest to the proton-proton collision point. It is designed for a 2 Tesla strong axial magnetic field at the collision point, while its thin-walled construction of 0.66 radiation lengths avoids degradation of energy measurements in the outer calorimeters. The solenoid and calorimeter have been integrated in their common cryostat, cooled down and tested on-surface. We review the on-surface set-up and report the performance test results.
\end{abstract}

Index Terms-Detector, magnet, quench results.

\section{INTRODUCTION}

$\mathbf{T}$ HE ATLAS collaboration is preparing a general purpose detector set-up for experiments with proton-proton collisions at the Large Hadron Collider (LHC) at CERN. The detector consists of four main parts: inner detector trackers, electromagnetic calorimeter, hadron calorimeter and muon spectrometer. The superconducting solenoid is the central part of a large magnet system. It provides the magnetic field for the crucial momentum measurements of charged particle tracks in the inner detector. It is also of vital importance that the magnet does not disturb the energy measurements of particles in the calorimeter located outside the magnet. A solution was found in a thin-walled superconducting solenoid, indirectly cooled by circulation of two-phase helium. The solenoid shares its cryostat with the electro-magnetic calorimeter, which, filled with liquid argon, serves also as thermal shield for the solenoid. With this arrangement the transparency for particles passing the solenoid into the calorimeter is $0.66 X_{0}$ (radiation lengths). Its major features are described elsewhere [1], [2]. The main parameters are listed in Table I.

The solenoid system is being developed in collaboration between KEK, CERN and Toshiba. After fabrication in Japan all equipment was shipped to CERN where it has been integrated with the barrel cryostat. The realization has been challenging due to its tight integration with the calorimeter. Proximity cryogenics and chimney were successfully commissioned last year [3].

Manuscript received October 4, 2004.

R. J. M. Y. Ruber, L. Deront, F. Haug, G. Olesen, O. V. Pavlov, M. Pezzetti, O. Pirotte, E. Sbrissa, and H. H. J. ten Kate are with the CERN, CH-1211 Geneva 23, Switzerland (e-mail: ruber@cern.ch).

Y. Makida, Y. Doi, T. Haruyama, M. Kawai, T. Kondo, Y. Kondo and A. Yamamoto are with the KEK, Tsukuba, 305-0801 Japan.

S. Mizumaki is with the Toshiba Co., Tsurumi, Yokohama, 230-0045 Japan.

Digital Object Identifier 10.1109/TASC.2005.849571
TABLE I

MAIN PARAMETERS OF THE SUPERCONDUCTING SOLENOID

\begin{tabular}{ll}
\hline \hline Central Field & $2.0 \mathrm{~T}$ \\
Operating Current & $7600 \mathrm{~A}$ \\
Coil Size & $5.3 \mathrm{~m}(\mathrm{l}) \times 2.4 \mathrm{~m}(\Phi) \times 45 \mathrm{~mm}(\mathrm{t})$ \\
Wall Thickness & $0.66 \mathrm{X}_{0}$ \\
Stored Energy & $39 \mathrm{MJ}$ \\
Inductance & $1.35 \mathrm{H}(1.27 \mathrm{H}$ for air core $)$ \\
Conductor Size & $30 \times 4.25 \mathrm{~mm}^{2}$ \\
Conductor Area Ratio & $1: 0.9: 14(\mathrm{NbTi}: \mathrm{Cu}: \mathrm{Al})$ \\
$\mathrm{I}_{c}$ at $5 \mathrm{~T}, 4.2 \mathrm{~K}$ & $22 \mathrm{kA}$ \\
Conductor RRR & $>500$ \\
\hline \hline
\end{tabular}

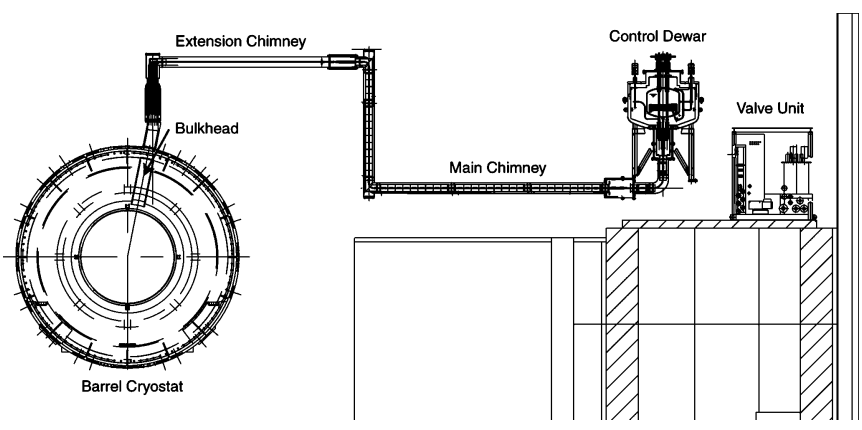

Fig. 1. Layout of the on-surface test set-up.

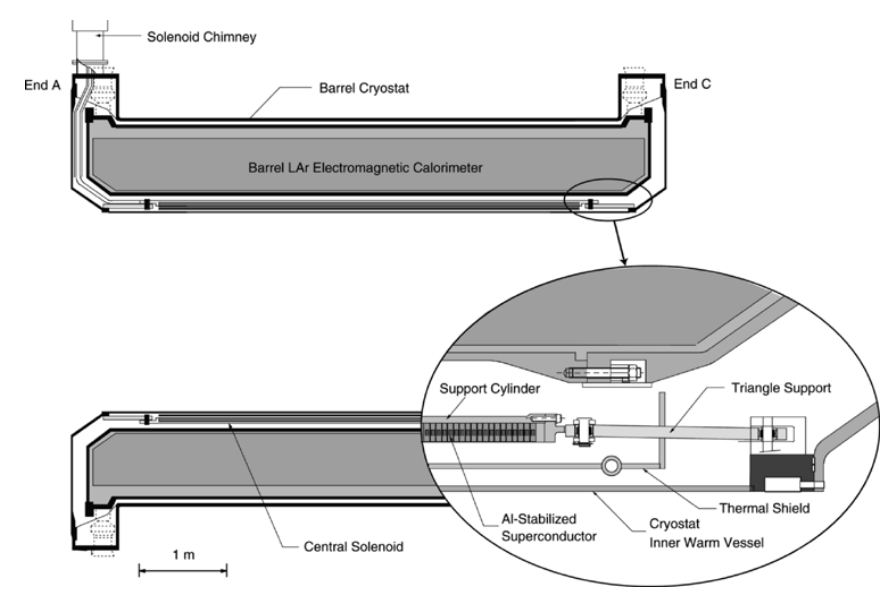

Fig. 2. Cross-section of the barrel cryostat with solenoid and calorimeter.

\section{ON-SURFACE INSTALLATION}

Fig. 1 presents the on-surface test set-up while in Fig. 2 is shown a detailed cross section of the barrel cryostat with solenoid and calorimeter. The solenoid coil is mounted on the inner warm vessel of the cryostat by 23 triangular glass fiber supports. The side of the coil toward the chimney is fixed while the opposite side can slide in axial direction [4]. After installation of the calorimeter wheels into the cryostat, the solenoid and inner 


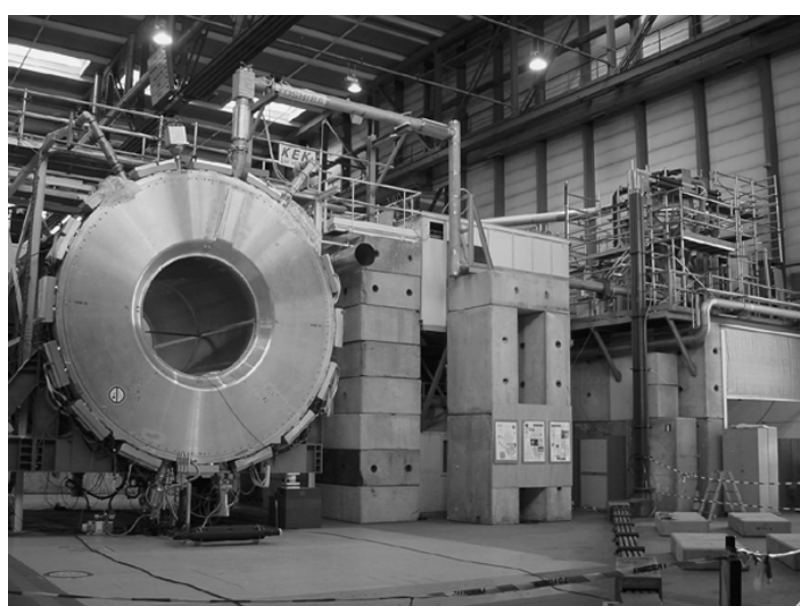

Fig. 3. The on-surface test set-up.

warm vessel where slided into position guided by a rails system. Then the superconducting bus-lines, consisting of a double pair of conductors, and the solenoid and thermal shield cooling lines were built-up in the bulkhead of the cryostat. After passing a feedthrough at an $11.25^{\circ}$ angle from the cryostat top, a joint was made to the chimney interconnecting solenoid and proximity cryogenics consisting of a control dewar and valve unit. The complete on-surface test set-up is shown in Fig. 3.

For the installation in the final ATLAS detector set-up, the proximity cryogenics will be located on top of the cryostat. Due to height limitations for the on-surface test set-up it was mounted to the side of the cryostat. Hence the interconnecting chimney was mounted in a horizontal position instead of vertically. For this the chimney was extended by $5.4 \mathrm{~m}$ in the horizontal direction; this is the extension chimney as marked in Fig. 1.

\section{MONITORING AND SAFETY SYSTEM}

The solenoid system is equipped with more than two hundred sensors to monitor its thermal, electrical and mechanical behavior. In addition there are five electrical heaters for quench initiation and protection on the coil and another five on the superconducting bus-line inside the cryostat and extension chimney.

The magnet safety system (MSS) used in the test was largely the same as the system to be used in the final ATLAS underground installation. The MSS monitors voltage signals, temperatures and superconducting quench detectors (SQD) on the coil, the bus-lines in the chimney and the current leads. These signals are used to indicate a failure and then trigger a slow dump (SD) or a quench condition of the magnet system and then trigger a fast dump (FD). In slow dump the MSS will switch off the magnet power supply and open the main breakers. In fast dump the MSS will react the same way, but fire the five quench protection heaters (QPHT) of the coil as well.

In addition MSS was monitoring the signal of pick-up coils installed at both ends of the solenoid. This signal was not yet used in signalling a fault condition, but the pick-up coil signal is fast and reliable in indicating a quench of the solenoid. The pick-up coil signal is subtracted from the total solenoid voltage.

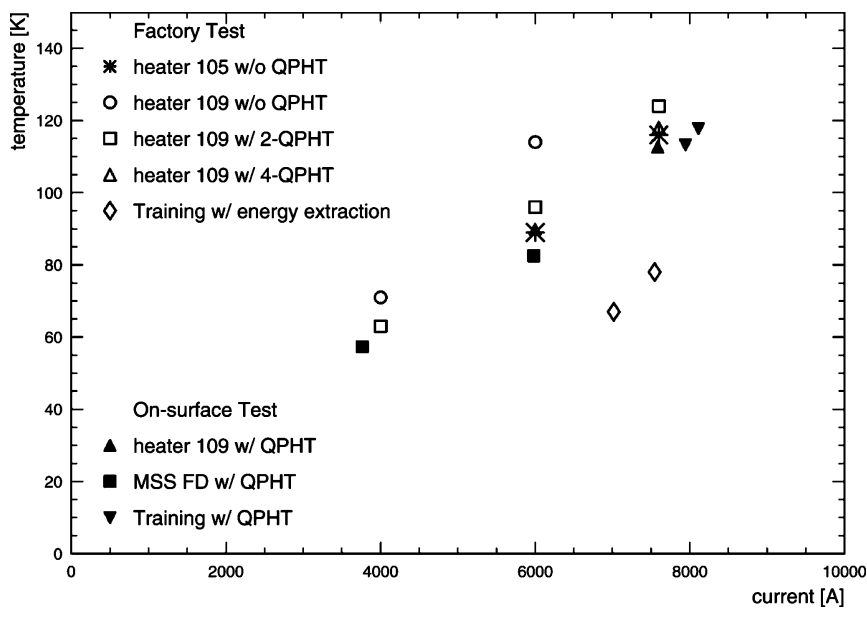

Fig. 4. Peak temperature of the coil winding after a quench.

This way the signal is neither di/dt sensitive during ramping nor does it react to external stray fields.

The monitoring was split in a fast and slow system. The slow monitoring was done by the magnet control system (MCS), a standardized control system for all LHC experiments. Like MSS, the MCS was largely similar to the final underground installation. Also the solenoid on-surface test provided the first operational test of its design. For the fast monitoring a data acquisition system with a FIFO memory was used. It consisted of a 16 bit $200 \mathrm{kHz}$ ADC configured to monitor 64 channels at $2 \mathrm{kHz}$. It was triggered by an MSS FD signal storing $10 \mathrm{~s}$ worth of pre-trigger data and 120 s post-trigger.

\section{PERformance Test}

The two main purposes of the on-surface test where to verify operation of the solenoid at nominal field and study the intrinsic safety of the design. In the ATLAS detector set-up the nominal operational field strength of the solenoid is $2.0 \mathrm{~T}$ at a current of $7.6 \mathrm{kA}$. In the air-core on-surface test set-up, a current of $8.0 \mathrm{kA}$ is required to reach the $2.0 \mathrm{~T}$ field strength. The maximum applied test current was $8.136 \mathrm{kA}$, close to the limit of the power system. Previously, during the reception test in Japan, the coil was excited up to $8.4 \mathrm{kA}$ to prove it has sufficient operating margin. The test repeated here has aimed at validating the system and verifying the stability of the solenoid after transport and installation in the cryostat.

\section{A. Quench Protection and Normal Zone Propagation}

Quench initiation was done with one of the five electric heaters on the coil winding, while the remaining four heaters were connected as quench protection heaters (QPHT) to MSS for a smooth temperature distribution after the quench. Quench initiation in the chimney was done by a heater in the middle of the extension chimney while all of the five coil heaters were connected as QPHT to MSS. Four quenches were initiated in the coil, at 4, 6 and $7.6 \mathrm{kA}$, and two in the chimney, both $6 \mathrm{kA}$, while there were also two training quenches at 7.9 and $8.1 \mathrm{kA}$. Fig. 4 presents the peak coil winding temperature after a quench. It combines the data from the factory test [5] and the present on-surface test. The chimney bus-line temperature went 


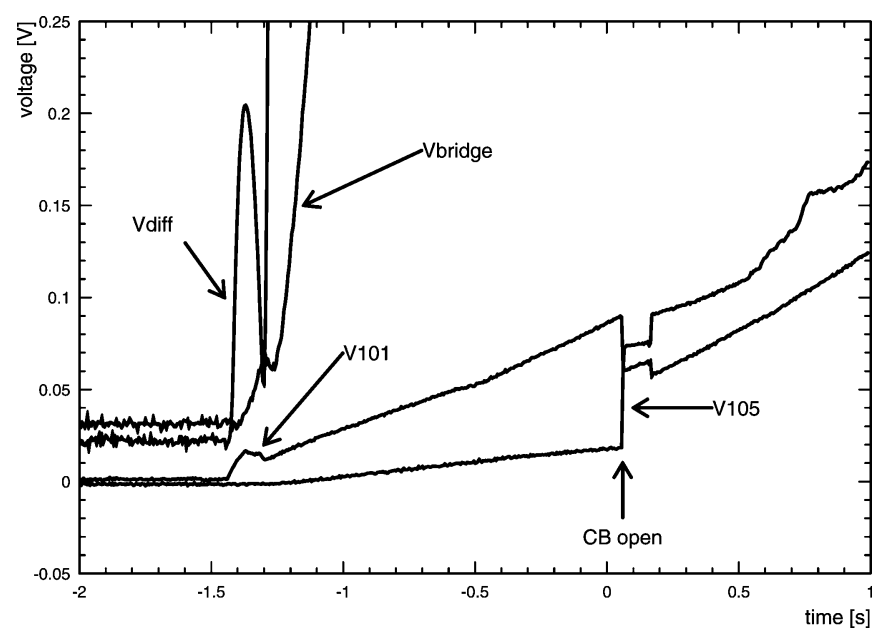

Fig. 5. Measured voltage during a quench. V101 and V105 are voltage taps at the coil. $V_{\text {diff }}$ and $V_{\text {bridge }}$ are the differential and bridge voltages from MSS.

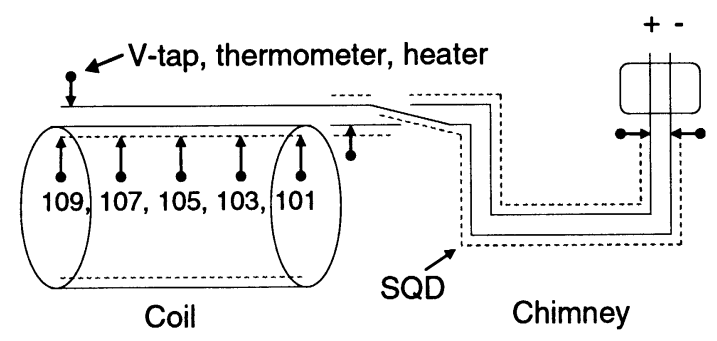

Fig. 6. Layout of the main instrumentation of the coil and chimney.

up to a maximum of $21 \mathrm{~K}$ both during a quench of the coil or a direct quench of the chimney itself af $6 \mathrm{kA}$.

There is a rapid reaction of coil and MSS to the firing of a heater. Within $0.2 \mathrm{~s}$ after the end $\mathrm{C}$ heater is fired (with 9.5 $\mathrm{W}$ during $8.4 \mathrm{~s}$, at $7.6 \mathrm{kA}$ ), the voltage of the coil starts rising. MSS has a $1 \mathrm{~s}$ filter on the input signals, and signals a quench detection in $1.59 \mathrm{~s}$ after the firing of the heater. The two superconducting quench detectors (SQD) which run along the top and bottom of the coil signal a complete transition to normal in $3 \mathrm{~s}$ after MSS triggered the fast dump (FD) and fired the quench protection heaters (QPHT), or $5 \mathrm{~s}$ after starting the quench initiation. The current was down to zero in approximately $70 \mathrm{~s}$.

In Fig. 5 the measured voltages are plotted versus the time. The location of the voltage taps on the coil is shown in Fig. 6 . They are installed at each quarter of the coil length. MSS detects a quench at $\mathrm{t}=0 \mathrm{~s}$ and then opens the electric circuit breaker (CB) and fires the QPHT's. The differential voltage $V_{\text {diff }}$ of a pick-up coil with the total coil voltage is plotted as well as the bridge voltage $\mathrm{V}_{\text {bridge }}$ between voltage taps 101, 107 and 109. The fast response of the differential voltage signal is clearly visible.

The normal zone propagation velocity can be estimated from the signals of the different voltage taps. In the chimney a longitudinal propagation velocity of $2.4 \pm 0.6 \mathrm{~m} / \mathrm{s}$ was measured. This corresponds to the estimated velocity with an uniform current distribution over the aluminum stabilizer. The rapid reaction of MSS to quench detection, and its firing of the QPHTs, prevented a study of the normal zone propagation velocity in the coil.

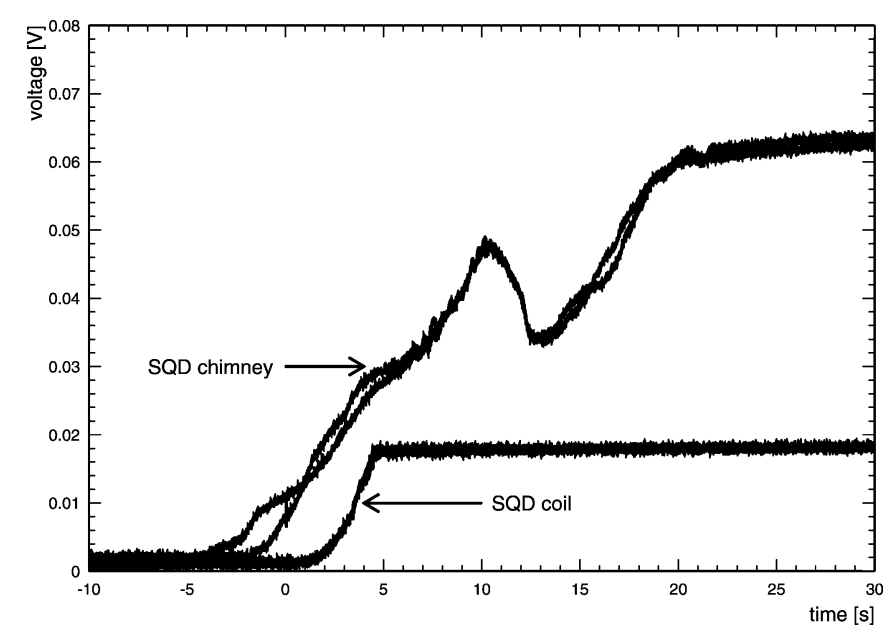

Fig. 7. SQD signals during a quench in the chimney.

Fig. 7 displays the evolution of the SQD signals during a heater induced quench of the chimney. After the MSS detects the quench, the QPHT at the coil are fired. The SQD signals indicate a temporary recooling of the chimney by the cold gas front produced during the coil quench.

The gradient between the hot spot temperature of the coil winding and support cylinder is typically in the order of $25 \mathrm{~K}$ after a quench at $8 \mathrm{kA}$. The temperature gradient along the coil is around $35 \mathrm{~K}$ from the initial quench location to the opposite end of the coil.

\section{B. Heat Load}

After closure of the cryostat the calorimeter was cooled down first. It reached a final temperature of $89 \pm 1 \mathrm{~K}$ after filling with liquid argon, which is used as particle detection medium. The temperature of the solenoid did not decrease more than 20 degrees: from a room temperature of $292 \mathrm{~K}$ to $275 \pm 1 \mathrm{~K}$ and $284 \pm 1 \mathrm{~K}$ for the thermal shield.

The static thermal load on the solenoid can be estimated from the temperature gradient during warm-up. In the first phase of the warm-up the thermal shield and detector temperatures were constant $(78.5 \pm 0.1 \mathrm{~K}$ and $89 \pm 1 \mathrm{~K}$ respectively). This indicates a heat load on the coil of $7.8 \pm 0.9 \mathrm{~W}$. This is better than the $10.5 \mathrm{~W}$ static thermal load measured during the factory test [6]. An improvement of the present installation is that between solenoid and outer thermal shield (the calorimeter) the amount of multilayer superinsulation (MLI) has almost doubled.

Cooling mass flow stop studies were done at currents of $4 \mathrm{kA}$. It was possible to complete a slow ramp down (SD) of the solenoid in 41 minutes before a temperature rise of the magnet system triggered MSS into a FD. In the final underground installation a different run-down unit will be used that enables a SD from $7.6 \mathrm{kA}$ to zero in less than half an hour.

Cooling of the current leads worked well and ice build-up at the top of the leads was prevented by a fan forcing an ambient air flow along the top of the leads.

\section{Mechanical Behavior}

The coil position is monitored by potentiometers mounted in axial and circumferential direction at both ends of the coil. At 


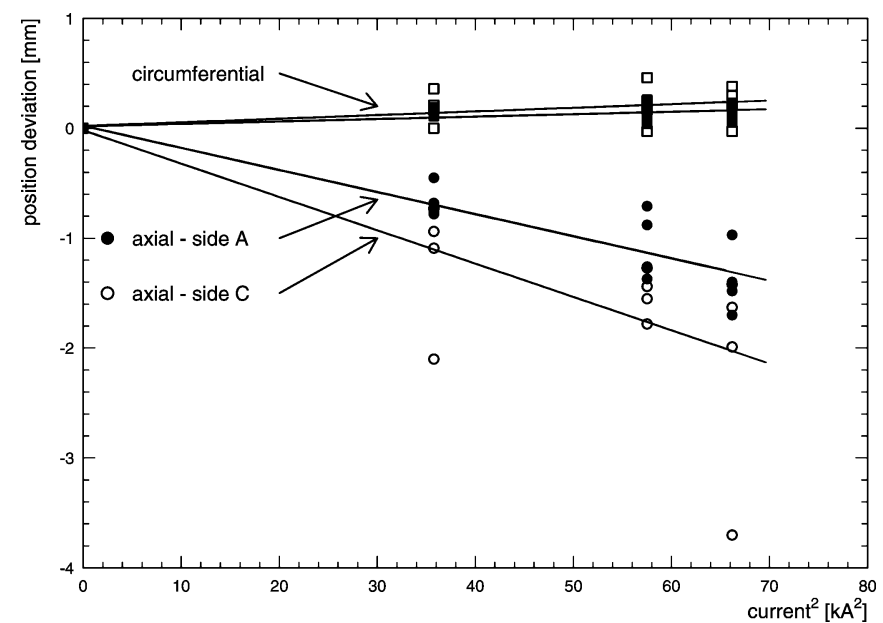

Fig. 8. Position measurement during excitation.

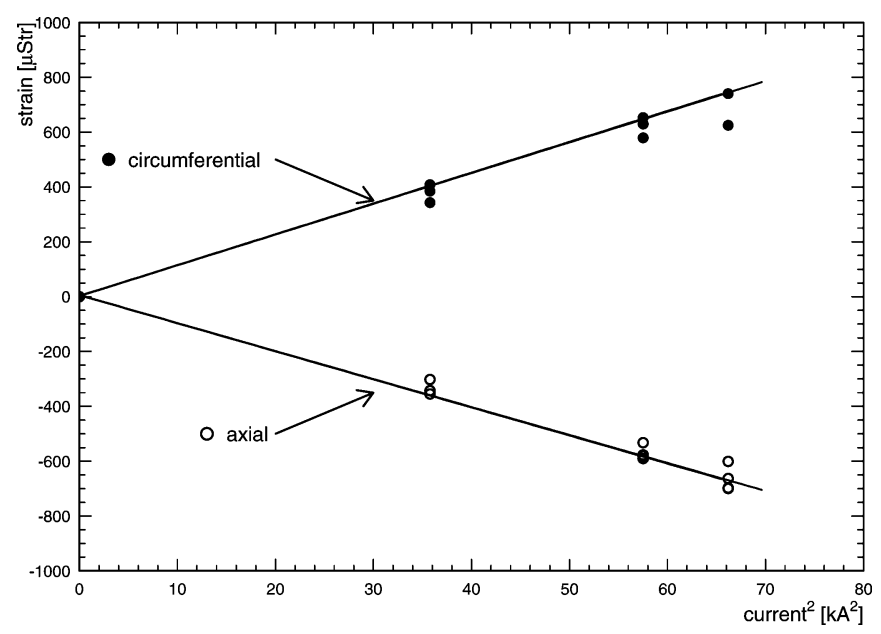

Fig. 9. Support cylinder strain during excitation.

end $\mathrm{C}$ the supports are allowed to slide freely in axial direction while the support movement at end $\mathrm{A}$ is limited to $<0.5 \mathrm{~mm}$ due to the chimney connection. The measured axial displacement during cool down averaged $-22.0 \pm 0.9 \mathrm{~mm}$, which corresponds to $0.39 \%$ of the $5698.2 \pm 0.8 \mathrm{~mm}$ coil length. The coil length (winding including end flanges) was surveyed before insertion in the cryostat. It confirms a $1.8 \mathrm{~mm}$ permanent shrinkage after the factory test of the coil.

Strain gauges were mounted at the top and bottom of the support cylinder at similar locations as for the voltage taps as indicated in Fig. 6. Measured strain is approximately $700 \mu \mathrm{Str}$ in both axial and circumferential direction during excitation to 8.1 kA, see Fig. 9. This circumferential strain level indicates a hoop stress of $51 \mathrm{MPa}$ as expected from the design values [1]. As anticipated, the measured strain is highest in the center of the coil and decreases toward the ends of the coil. Near the end flanges the circumferential strain is $250 \mu$ Str suggesting a hoop stress of $18 \mathrm{MPa}$.

Fig. 8 presents the position sensor read-out versus the square of the excitation current. Axial position sensors indicate a change of $-3.58 \pm 1.35 \mathrm{~mm}$ at $8.1 \mathrm{kA}$, circumferential sensors indicate $0.3 \pm 0.2 \mathrm{~mm}$. This agrees well to the measured strain as $\epsilon_{c}=250 \mu \mathrm{Str}$ would give a $\Delta \mathrm{r}=0.31 \mathrm{~mm}$. And the axial strain $\epsilon_{a}=-699 \mu \mathrm{Str}$ as measured at the center would give a $\Delta \mathrm{z}=-3.98 \mathrm{~mm}$ if it was uniform over the coil length and $\epsilon_{a}=-494 \mu \mathrm{Str}$ at the end flanges gives $\Delta \mathrm{z}=-2.81 \mathrm{~mm}$.

\section{CONCLUSIONS}

The on-surface test verified the operation of the ATLAS superconducting solenoid after transport to CERN and integration in the barrel cryostat. Detailed studies of the quenching behavior have confirmed safe operation at currents up to $7 \%$ above nominal. The magnet safety system has been validated for use in all LHC experiments. Its combination of superconducting quench detectors, pick-up coils and quench protection heaters makes an excellent protection system which also limits the peak temperature after quench. Installation in the 100 meters deep detector cavern will now be started.

\section{ACKNOWLEDGMENT}

The authors would like to thank their colleagues from KEK, Toshiba, CERN and ATLAS for their extensive efforts during the integration and test. R. Ruber is grateful to Uppsala University for supporting his work.

\section{REFERENCES}

[1] A. Yamamoto et al., "Design and development of the ATLAS central solenoid magnet," IEEE Trans. Appl. Superconduct., vol. 9, p. 852, 1999.

[2] _ - "Progress in ATLAS central solenoid magnet," IEEE Trans. Appl. Superconduct., vol. 10, p. 353, 2000.

[3] R. J. M. Y. Ruber et al., "On-surface integration and test of the ATLAS central solenoid and its proximity cryogenics," IEEE Trans. Appl. Superconduct., vol. 14, p. 500, 2004.

[4] S. Mizumaki et al., "Fabrication and mechanical performance of the ATLAS central solenoid," IEEE Trans. Appl. Superconduct., vol. 12, p. 416, 2002.

[5] Y. Makida et al., "Quench protection and safety of the ATLAS central solenoid," IEEE Trans. Appl. Superconduct., vol. 12, p. 407, 2002.

[6] Y. Doi et al., "Performance of a proximity cryogenic system for the ATLAS central solenoid magnet," in Advances in Cryogenic Engineering: Proceedings of the Cryogenic Engineering Conference-CEC Volume 47, S. Breon et al., Eds., American Institute of Physics, 2002, p. 47. 